\title{
Le conflit conceptuel : de la grammaire aux métaphores
}

\section{Conceptual conflict : from grammar to metaphors}

\author{
Marco Fasciolo \\ Sorbonne Université EA-4509 STIH \\ Franck Neveu \\ Sorbonne Université EA-4509 STIH
}

\section{POUR UNE TOPOGRAPHIE DU CONFLIT}

Ce numéro de Langue Française est consacré à la notion de conflit - plus particulièrement au 'conflit conceptuel' - et prend appui, notamment, sur les travaux de Michele Prandi (1987, 1992, 2004 et 2017).

De manière générale, on peut parler de conflit, dans la langue, chaque fois que deux structures s'opposent sur une même unité. La généralité de cette formulation rend manifeste le privilège épistémologique de la notion de conflit. Quand des structures de la langue se superposent les unes aux autres, elles tendent à rester inaperçues; quand elles divergent, en revanche, ces mêmes structures se révèlent, en s'offrant ainsi à l'observation. Ce constat, dans sa formulation, reste cependant extrêmement abstrait et s'applique à tous les niveaux de réalisation de la langue.

Une première approche de la question consistera donc à distinguer différentes formes de conflit.

\subsection{Conflits et codage}

L'adjectif italien cortissimo signifie « très court », mais, en tant que mot, il est « long» (quatre syllabes): il y a donc un conflit entre son sens et sa taille. Le mot français jour renvoie à la clarté et à la lumière, mais, comme Mallarmé l'a souligné, sa voyelle $/ \mathrm{u} /$ est sombre. Il y a donc un conflit entre sa signification et les connotations liées au phonème /u/. Considérons les vers suivants :

1. Je ne te cache pas que j'aime aussi les bêtes ; Cela t'amuse et moi cela m'instruit; je sens

Que ce n'est pas pour rien qu'en ces farouches têtes

Dieu met le clair-obscur des grands bois frémissants 


\section{(V. Hugo, A Jeanne)}

Le rythme naturel du discours regroupe le verbe et son objet direct dans une même unité 'de discours', mais l'enjambement entre les vers 2 et 3 les sépare. Il y a donc un conflit entre la prosodie naturelle et le rythme artificiel crée par le vers. Considérons le poème suivant :

2. La faim le froid la solitude

Qui se méfient des asiles

Du blé fiévreux des morts.

(P. Eluard, La toile blanche)

L'action de se méfier présuppose un premier argument humain, mais la faim, le froid et la solitude sont des noms abstraits. Il y a donc un conflit conceptuel produit par la structure argumentale de se méfier et le référent du sujet de la relative.

Si les exemples précédents illustrent tous des conflits, on peut néanmoins établir une distinction majeure entre eux. Dans les deux premiers exemples, le conflit ne concerne pas une fonction linguistique. D'un côté, cela est évident pour la taille d'un mot ; de l'autre côté, s'il est vrai qu'un phonème remplit une fonction distinctive, il est également vrai que ce n'est pas cette fonction qui est valorisée dans l'exemple de Mallarmé (il s'agit plutôt d'une caractéristique accessoire de la substance phonique). Dans les autres cas, en revanche, le conflit concerne une fonction linguistique. La prosodie organise le flux discursif en unités communicatives au moyen de la distribution des pauses et des accents. Dans le texte de Victor Hugo, ces mêmes moyens sont valorisés pour produire un enjambement. Le premier argument du prédicat sémantique d'une phrase, en général, est saturé par une expression nominale au moyen de la fonction de sujet. Dans le poème d'Eluard, ce moyen est valorisé pour créer une connexion conceptuelle incohérente, qui pourra aboutir sur une métonymie ou une métaphore.

Nous venons de distinguer deux grandes espèces de conflits. D'un côté, il existe des conflits périphériques par rapport à la grammaire, parce qu'ils ne concernent pas ses ressources de codage. De l'autre, il y a des conflits qui se situent au centre de la grammaire, parce qu'ils concernent précisément ces ressources. Les figures du plan de l'expression sont à cheval entre ces deux espèces de conflit : la paronomase fait partie de la première, alors que l'enjambement fait déjà partie de la seconde (Prandi 2017:14). Les figures du plan du contenu, au contraire, se basent toutes sur un conflit relevant du code linguistique.

Puisque la paronomase n'est pas déclenchée par un conflit mettant en jeu des fonctions linguistiques, elle peut se révéler cruciale pour la description d'un texte contingent, mais elle ne nous dit rien à propos de la grammaire. En revanche, puisque l'enjambement et la métaphore reposent sur un conflit au niveau du code linguistique, ils peuvent mettre en lumière le fonctionnement de la grammaire. Le conflit à la base de l'enjambement révèle les mêmes structures à l'œuvre dans la prosodie. Le conflit à la base de la métaphore révèle les mêmes structures à l'œuvre dans l'idéation des procès complexes. 


\subsection{Conflit conceptuel vs. Coercition}

Nous nous intéressons aux conflits concernant des ressources de codage et, en particulier, à ceux qui se situent sur le plan du contenu. Le panorama est ici très varié.

Imaginons que, face à un rassemblement de forces de l'ordre, un manifestant s'exclame :

\section{Il y a du policier ici !}

Dans la mesure où cet exemple est perçu comme marqué, on peut affirmer que la matrice de détermination massive est transférée sur un nom pour lequel on s'attendrait plutôt à l'expression d'une détermination comptable. Considérons le passage suivant :

4. Enfin le navire partit ; et les deux berges, peuplées de magasins, de chantiers et d'usines, filèrent [...]

(Éducation Sentimentale, Flaubert)

Le verbe filer a une Aktionsart durative, mais l'Aspect perfectif du passé simple lui impose un formatage ponctuel et accompli. Considérons encore un exemple banal comme :

5. J'ai ouvert un vin de 2010.

Ici, on pourrait signaler une incompatibilité entre les restrictions du verbe ouvrir (impliquant un objet solide comme second argument) et le fait que le vin est un aliment liquide.

Les phénomènes précédents sont bien connus et ils ont fait l'objet d'une abondante littérature (Moens \& Steedman 1988, Nicolas 2002, Cruse 1986, Kleiber 1999, Pusteyovsky \& Jezek 2008, etc.), à laquelle correspond une abondance d'étiquettes: " type shifting », « modulation», " coercition», " métonymie intégrée », etc. Tout en étant conscients de leur hétérogénéité - et de leur probable irréductibilité - nous suivons une attitude répandue en les regroupant tous sous le chapeau de « coercition ».

Or, si la coercition est un ensemble de stratégies d'accommodation déclenchées par une quelconque forme de conflit, il faut tracer une ligne de démarcation nette entre cette forme de conflit et les conflits conceptuels prototypiques. À cet égard, l'exemple (5) appelle deux remarques. Premièrement, au-delà d'un cours de grammaire, il serait extrêmement pédant de chercher une incompatibilité entre ouvrir et vin, alors que ce serait tout à fait naturel pour le verbe hurler et le nom la rue dans : La rue assourdissante autour de moi hurlait (A une passante, Baudelaire). Deuxièmement, et surtout, la première des coordinations suivantes n'est pas un zeugme, mais la seconde en est un:

6. J'ai ouvert un vin de 2010 et je l'ai versé par terre. 
7. *La rue assourdissante autour de moi hurlait et elle était pleine de dos-d'ânes.

Par ailleurs, dans un vin ouvert, ouvert n'indique pas une caractéristique de la bouteille où se trouve le vin, mais le vin lui-même; inversement, dans une rue en révolte, en révolte indique bien une caractéristique des gens qui se trouvent dans la rue, et non de la rue elle-même. Ces constats suggèrent non seulement que la coercition n'implique ni de changement référentiel, ni de polysémie (Prandi 2017 :194-195), mais qu'ils indiquent aussi qu'à l'intérieur de la phrase La rue [...] hurlait il y a bien un conflit conceptuel potentiel, alors qu'à l'intérieur de la phrase J'ai ouvert un vin de 2010 il n'y en a guère.

\subsection{Conflit conceptuel vs. Interprétation non-littérale}

À l'intérieur du plan du contenu, la coercition délimite le domaine du conflit conceptuel, pour ainsi dire, vers le bas. Le conflit conceptuel, cependant, doit être également délimité vers le haut, en l'opposant aux conflits ayant lieu au-delà du plan du contenu, dans l'espace de l'interprétation.

Imaginons une petite scène familiale :

8. Mari : Qui va récupérer les enfants à l'école demain ? Femme : Chéri, demain je vais à la danse.

Dans le dialogue (8), il y a un décalage entre la signification de (8) et le message communiqué (demain, ce n'est pas moi, mais toi, qui iras récupérer les enfants). Nous sommes confrontés au type de conflit à l'origine des interprétations nonlittérales.

Rappelons que des propriétés telles que contradictoire, cohérent ou incohérent s'appliquent au niveau symbolique de la signification, alors que littéral et nonlittéral s'appliquent à la relation entre ce niveau et le niveau pragmatique du message (cf. Prandi 2000). Or, ces niveaux peuvent accueillir des conflits indépendants entre eux.

Il peut y avoir un conflit au niveau de l'interprétation, mais non du contenu : c'est justement le cas de (8). Il peut y avoir un conflit au niveau du contenu, mais non de l'interprétation :

9. Une Montagne en mal d'enfant

Jetait une clameur si haute,

Que chacun, au bruit accourant,

Crut qu'elle accoucherait, sans faute,

D'une cité plus grosse que Paris ;

Elle accoucha d'une souris

(La montagne qui accouche, La Fontaine)

Par rapport à l'ontologie fictive des fables, la montagne gémit et accouche vraiment : des significations conceptuellement conflictuelles aboutissent donc à une 
interprétation littérale, non-conflictuelle (Prandi 2004:15). Il peut y avoir un conflit aux deux niveaux en même temps :

10. Les gros bureaux bouffis traînent leurs grosses dames ( $A$ la musique, Rimbaud).

Le premier conflit est interne à la signification : le premier argument de traîner est une force, mais les bureaux sont des lieux. Le second conflit est externe au précédent et consiste dans le décalage entre cette signification conflictuelle et la critique à l'encontre du bourgeois que Rimbaud veut communiquer avec ce même vers.

Souligner l'indépendance réciproque entre ces deux niveaux de conflit permet de distinguer entre deux familles de tropes. D'un côté, l'allégorie ou l'antiphrase (les tropes « diffus ", cf. Fontanier 1821) sont définis par la présence du conflit au niveau de l'interprétation : ils ont donc la même nature que l'exemple (8). De l'autre côté, les métaphores vives et les métonymies (les tropes en " un seul mot», cf. Fontanier 1821) peuvent contenir les deux conflits: elles ne sont donc pas réductibles à des formes de non-littéralité. Nous nous intéressons en particulier à ces dernières.

Remarquons que, avant d'établir si (10) doit recevoir une interprétation métonymique (comme c'est le cas) ou métaphorique (comme c'est en principe possible), on peut se demander si ces tropes se situent au niveau du conflit interne à la signification, ou bien au niveau du conflit concernant le rapport entre signification et message. En fait, ils se situent entre les deux. Au-delà de la signification, il y a une inférence abductive qui amène au message, mais qui présuppose le fait d'avoir déjà décidé s'il s'agit d'une métonymie ou d'une métaphore. Au niveau de la signification, il n'y a rien d'autre qu'un conflit conceptuel qui attend d'être interprété en tant que métonymie ou en tant que métaphore. Comme Prandi (2004:347) le souligne : "The meaning of a metaphorical expression is not any solution the conflict is open towards, but the conceptual conflict itself».

Cela illustre la position stratégique de la notion de conflit conceptuel.

\subsection{Le conflit conceptuel parmi les conflits du contenu}

Si nous excluons d'une part les phénomènes de coercition et, de l'autre, les conflits de la non-littéralité, il nous reste une zone, interne au plan du contenu, qui est très peu analysée. En témoigne la non-systématicité des étiquettes utilisées par les auteurs qui se sont penchés sur les tropes du contenu : anomalie sémantique, rupture avec la logique, incompatibilité, contradiction, incongruence, etc. (Fasciolo $\&$ Rossi 2016:6). Bien que ces étiquettes dessinent un champ sémantique centré implicitement sur l'idée de conflit, cette dernière est demeurée non analysée car l'attention s'est focalisée sur ses effets plutôt que sur la nature du conflit lui-même.

Prandi (2017:ch.3) propose une typologie des conflits du contenu :

11. J'aime et je hais (Odi et Amo, Catulle)

12. Ces vendeurs aboient. 
13. De l'océan de l'air les poissons emplumés (Chevreau, repris par Genette 1966:§1).

14. Mon dentiste est un boucher.

15. La lune s'attristait (Apparition, Mallarmé).

L'exemple (11) est une contradiction. Une contradiction découle de la conjonction entre deux prédications opposées, qui sont conceptuellement cohérentes (je désigne bien un être humain capable d'éprouver des sentiments) et lexicalement correctes. Ce type de conflit est à la base de l'oxymore.

L'exemple (12) est un conflit lexical. Un conflit lexical ne brise pas notre ontologie naturelle partagée (des vendeurs sont bien des êtres animés pouvant émettre des cris), mais une solidarité syntagmatique: aboyer est le mot que le lexique prévoit les cris des chiens et non des personnes.

L'exemple (13) est un conflit cognitif. Un conflit cognitif ne brise pas notre ontologie naturelle partagée (des poissons emplumés, comme des poissons volants ou des poissons avec des poumons, restent des animaux) et ne falsifie non plus nos connaissances encyclopédiques. En revanche, il aboutit à une instance marginale (fictive ou réelle) d'un prototype partagé. La notion de prototype est donc bel et bien une stratégie pour gérer des conflits cognitifs dans les limites de la cohérence conceptuelle.

L'exemple (14) est un conflit empirique. Un conflit empirique, à la différence du cas précédent, falsifie ponctuellement notre expérience : il s'agit du « faux ».

Enfin, l'exemple (15) est un cas de conflit conceptuel. Un conflit conceptuel brise précisément l'ontologie naturelle présupposée par tous les précédents conflits (Prandi 2016, Fasciolo 2016, 2018). Une lune triste n'est pas un corps céleste non prototypique, mais ce n'est plus un corps céleste. C'est le type de conflit le plus profond.

\section{LE CONFLIT DANS LA LITTÉRATURE SPÉCIALISÉE}

La topographie esquissée sous $\S 1$, offre quelques pistes pour s'orienter dans la littérature qui a traité, plus ou moins directement, du conflit. En utilisant le conflit conceptuel comme point de repère, nous pouvons distinguer - très schématiquement - trois groupes de travaux. Certains travaux se situent en dessous du conflit conceptuel: il s'agit des contributions qui s'intéressent aux phénomènes de coercition, souvent dans le sillage de la sémantique générative (\$2.1.). D’autres travaux se situent au-dessus du conflit conceptuel : il s'agit de contributions qui s'intéressent au niveau pragmatique, dans le cadre de la théorie de la pertinence (cf. Sperber \& Wilson 1986) (\$2.2.). D'autres travaux encore se situent effectivement dans le même champ du conflit conceptuel (§2.3.) : nous pensons au débat autour de l'autonomie de la syntaxe qui a opposé la syntaxe générative des origines (Chomsky 1957) au tournant fonctionnel-cognitif (Fillmore 1968, 1977 ; Dik 1989, Langacker 1993, 2000). 


\subsection{En dessous du conflit conceptuel}

Le volume de Francis \& Michaelis (2003) met en avant la notion de mismatch (divergence), qui s'applique aux exemples de coercitions sous $\S 1.2$. La notion de divergence capture l'idée centrale du conflit. Cependant, dans la mesure où cette notion se limite à prolonger celle de coercition, elle en hérite les caractéristiques : à savoir, l'exclusion de tout véritable conflit conceptuel (cf. §1.2.).

Insistons sur un point. Il ne faut pas confondre la complexité ontologique d'un objet avec le conflit conceptuel : se déplacer sur les 'facettes' d'un objet polyédrique présuppose l'intégrité ontologique de cet objet. Si je critique et je déchire un livre ou si j'ouvre et je bois un vin, le livre et le vin restent ce qu'ils sont : leur identité n'est pas remise en question. En revanche, si je dis La salle rigole de moi, l'identité de la salle - qui n'est pas un être humain, mais un lieu - est menacée, et la métonymie est justement une stratégie pour contourner cette menace.

\subsection{Au-dessus du conflit conceptuel}

La théorie de la pertinence, quant à elle, a un grand mérite : elle a mis en valeur les interprétations non-littérales ou obliques des énoncés. Ce faisant, cependant, elle a fini par détourner l'attention du conflit interne au contenu aux conflits concernant l'interprétation (Prandi 2017:31). Ce n'est pas par hasard que, dans ce cadre, le trope prototypique est l'allégorie (Perrin 1996) et que la métaphore s'avère réduite à un cas d'interprétation non littérale parmi les autres. Ce faisant, cependant, on cache sa spécificité, qui découle de la présence d'un conflit aux deux niveaux : non seulement au niveau de l'interprétation, mais également au niveau de la signification (cf. $\S 1.3$.$) .$

Sans nier l'apport de ces approches, nous voudrions donc ré-déplacer l'attention sur ce dernier type de conflit.

\subsection{Dans le champ du conflit conceptuel}

Le confit conceptuel illustré par (15) (La lune s'attristait) est le lieu naturel où discuter la question de l'iconicité ou de l'autonomie de la syntaxe : le noyau du célèbre exemple des idées vertes incolores dorment furieusement manifeste en effet précisément ce type de conflit. On pourrait donc s'attendre à ce que le conflit conceptuel ait été valorisé par les paradigmes de recherche qui se sont opposés, justement, sur cette question. Il n'en est pas ainsi. À cet égard, il est intéressant de jeter un regard épistémologique sur les sources de ces paradigmes.

La syntaxe formelle de Chosmky (1957) a comme arrière-plan philosophique la perspective néopositiviste de Carnap (1932), qui se propose d'éliminer les énoncés conceptuellement incohérents. Ce fait a produit un paradoxe, brillamment remarqué par Jakobson (1966). D’un côté, le postulat théorique de Chosmky (1957) l'autonomie de la syntaxe - implique logiquement le conflit conceptuel : si la syntaxe est autonome par rapport aux structures conceptuelles ou sémantiques, alors elle doit générer des phrases conceptuellement cohérentes et incohérentes. De l'autre côté, à cause de cet arrière-plan néopositiviste, la possibilité de phrases 
conceptuellement incohérentes a été immédiatement perçue comme un problème (cf. Chomsky 1967). La conséquence en est que le paradigme de la syntaxe générative devient épistémologiquement contradictoire.

Le tournant fonctionnel et cognitif a pour initiateur Fillmore (1968) et pour précurseur Tesnière (1959). L'arrière-plan philosophique reside dans oeuvre de Wittgenstein (1921: §4.01) : «A proposition is a picture of reality. A proposition is a model of reality as we imagine it $»$. Le postulat théorique peut se résumer au fait que la syntaxe représente des structures conceptuelles indépendantes (Haiman 1985:2, Langacker 1993:465). Ce postulat exclut logiquement le conflit conceptuel. En effet, toute représentation de quelque chose ou perspective sur quelque chose peut être plus ou moins schématique, plus ou moins caricaturale, plus ou moins oblique, etc., mais - par définition - elle ne se conçoit pas sans ce quelque chose. Comme Dik (1997:8) l'écrit : « In this view, there is no room for something like an 'autonomous' syntax ». L'idée de construction (Goldberg 1995), pour sa part, peut être envisagée comme l'aboutissement du postulat précédent. Une construction n'est pas le résultat d'un processus d'idéation ou de composition, mais une sorte de signe, articulé, qui représente en bloc une signification. Comme Goldberg (1995:1) l'écrit : «Constructions themselves carry a meaning ». Or, entre un signe et sa signification, il n'y a aucun sens à parler de " conflit ».

Considérons encore le cas des métaphores conceptuelles. Certes, l'argent n'est pas un liquide, mais le concept étiqueté «l'argent EST un liquide » n'est pas créé par la langue : il est exprimé par la langue. Les métaphores conceptuelles excluent donc le conflit dans la mesure où elles reproduisent des concepts partagés. Lakoff \& Johnson (1980), par ailleurs, bannissent le conflit conceptuel de la création poétique car, selon eux, cette dernière se réduit au développement - élaboration - de réseaux de métaphores conceptuelles cohérentes ${ }^{1}$.

En somme, à la différence de la grammaire générative des origines, le postulat épistémologique du tournant cognitif - l'iconicité - est incompatible avec l'idée même de conflit conceptuel. Cela ne signifie pas que le conflit soit banni tout court, mais que son espace se réduit aux cas de non-prototypicité ou à des phénomènes de coercition.

Dans le domaine de la lexicographie issue d'une approche corpus driven, la notion d' "exploitation d'une norme " de Hanks (2013) mérite une attention particulière. Il s'agit d'un escamotage ingénieux, qui justifie les emplois les plus créatifs en tant qu'extensions analogiques de patterns d'usage ${ }^{2}$. Cette idée rappelle, mutatis mutandis, la notion d'écart de la tradition littéraire et rhétorique. Cette tradition, en effet, intègre la notion de conflit (cf. par exemple, Hallyn 1975, 1987) en l'envisageant comme transgression : à ce propos, nous renvoyons à l'école néorhétorique (Groupe $\mu$ 1970, Cohen 1979).

\footnotetext{
Dans le sillon cognitif, la problématique du conflit se manifeste dans la difficulté à expliquer les « métaphores délibérées » (Steen 2008, 2011).

La notion d'exploitation de Hanks (2013) a comme modèle l'exploitation des maximes conversationnelles de Grice. L'exploitation de ces dernières se situe au niveau du processus de communication d'un message ; l'exploitation d'un pattern au sens de Hanks (2013), en revanche, se situe au niveau de l'usage en corpus (qui devrait nous révéler la sémantique d'une certaine structure).
} 
Envisager le conflit en termes d'exploitation ou transgression d'une norme, cependant, a deux inconvénients. Le premier inconvénient est un risque : le risque que les étiquettes exploitation ou transgression fonctionnent comme des pierres tombales sur le conflit, en empêchant une analyse fine de ses différentes formes (cf. $\S 1.4)$. Le second inconvénient, en revanche, est un fait. Le point de référence par rapport auquel on mesure la transgression, ou le pattern qui est bafoué par les parlants les plus innovants, relève précisément du code linguistique. Cela présuppose que la fonction élective de ce dernier - et en particulier de la syntaxe n'est pas la production du conflit lui-même, qui en est justement une déviance (quoi que créative). Le conflit se révèle ainsi un phénomène intéressant, mais épistémologiquement négatif car le code linguistique, en soi, n'a pas la fonction intrinsèque de générer le conflit.

Ce présupposé, qui peut paraître raisonnable à première vue, est le point commun partagé aussi bien par les travaux linguistiques que littéraires. Dans ce numéro nous voudrions mettre à l'épreuve ce présupposé en envisageant le conflit conceptuel comme un phénomène positif.

\section{LE CONFLIT CONCEPTUEL EN TANT QUE PHÉNOMĖNE POSITIF}

La fonction élective des ressources grammaticales de codage - et notamment de la syntaxe - est de connecter des concepts atomiques dans des structures complexes. Envisager le conflit conceptuel en tant que phénomène positif - comme Prandi (1987, 1992, 2004 et 2017) le propose - signifie l'envisager non pas comme un effet accessoire de cette fonction, mais comme l'une de ses raisons d'être.

Une raison d'être de la syntaxe est sans doute l'instrumentalité au sens de Dik (1997:8) : dans ce cas, la connexion des concepts atomiques reproduit les relations naturelles entre ces concepts en leur donnant éventuellement une certaine perspective. Mais une autre raison d'être de la syntaxe est la créativité (cf. Prandi 2017:285) : connecter les concepts indépendamment de tout modèle cohérent donné au préalable. Le conflit conceptuel, qui est à l'origine des cas les plus intéressants de métaphores vives, est le produit de cette connexion.

En se focalisant sur l'idée de conflit, le présent numéro de Langue Française entre en dialogue, nous semble-t-il, avec le numéro 160 intitulé Figures et points de vue (Rabatel 2008). Les deux numéros, en effet, partagent l'idée que le discours figuré n'est pas un accident de la langue, mais bien le point d'observation privilégié pour décrire les processus de construction du sens. La différence concerne l'expression «construction du sens ». Dans le numéro précité, cette expression désigne la construction d'un "point de vue » au sens de Ducrot (1980); dans le présent numéro, en revanche, cette expression désigne la connexion de concepts atomiques dans des procès complexes. Dans un cas, la perspective est discursive et pragmatique; dans l'autre, la perspective est sémantique et syntaxique.

L'idée est que, dans une langue, l'on puisse identifier un noyau circonscrit de structures formelles dont la fonction n'est pas seulement de permettre l'expression de réseaux conceptuels cohérents, partagés, mais bien de faire rentrer ces réseaux en conflit. Autrement dit, le but de l'architecture $\mathrm{GN}^{\mathrm{SUJET}}+\mathrm{GV}$ n'est pas seulement 
d'exprimer un procès cohérent comme dans la phrase La lune brille, mais - surtout de construire un procès incohérent comme la phrase La lune rêve (Tristesses de la lune, Baudelaire).

Selon Prandi (2004, 2017), le noyau des structures formelles que nous venons de mentionner identifie un régime de codage « relationnel ». En français, ce régime comprend le sujet, le c.o.d, l'objet indirect (des verbes dits « di-transitifs ») et l'objet prépositionnel (des verbes dits «transitifs indirects »). L'existence d'un régime de codage relationnel et la possibilité de conflits conceptuels, par ailleurs, sont compatibles avec, et impliquent, l'existence d'un autre régime de codage, que Prandi $(2004,2017)$ appelle «ponctuel ». Ce dernier est effectivement gouverné par un principe iconique ou instrumental par rapport aux réseaux conceptuels partagés. Chaque phrase manifeste un équilibre variable entre ces deux formes de codage.

Une illustration plastique de ces régimes de codage est offerte par l'architecture de la phrase $\mathrm{GN}^{\mathrm{SUJET}}+\mathrm{GV}$ d'un côté et la structure du groupe nominal de l'autre. Observons l'exemple (16) :

\section{Le jeu des clous.}

L'exemple (16) pourrait être interprété comme signifiant un jeu auquel on joue avec des clous ${ }^{3}$. Dans ce cas, l'interprétation sémantique reproduit un réseau conceptuel cohérent et partagé : les clous sont des outils pouvant être intégrés dans une activité humaine en tant qu'instruments. Nous sommes dans un régime de codage ponctuel. Cela n'implique pas qu'en (16) tout conflit conceptuel soit exclu : sous la pression du co-texte ou avec un peu d'imagination nous pouvons bien forcer une interprétation incohérente selon laquelle ce sont les clous qui jouent ${ }^{4}$. Cependant, non seulement cette dernière n'est pas l'interprétation par défaut, mais la possibilité même d'une telle alternative - qui est un cas d'indétermination et non d'ambiguïté (cf. Prandi 2004:136) - prouve que la structure syntaxique $N$ de $N$ n'est pas capable d'imposer, de construire, des relations conceptuelles conflictuelles : elle exprime tout simplement des relations conceptuelles préalables (s'il y en a).

Or, une telle alternative est exclue dans le cadre de la phrase (17) :

\section{Les clous jouent.}

Ici, nous sommes dans un régime de codage relationnel. Encore une fois, cela n'implique pas que le conflit conceptuel ne puisse pas être contourné, atténué ou résolu, mais que ce conflit est bien construit ou imposé par la structure syntaxique de la phrase indépendamment des connexions naturelles entre les concepts. porte ce nom.

Il y a en effet un jeu pour enfants d'inspiration Montessori qui

Les deux lectures, par ailleurs, ne sont pas au même niveau. La lecture cohérente - en tant que par défaut - n'a pas besoin de la prise en compte du co-texte : elle relève donc de la sémantique. La lecture conflictuelle, en revanche, est déclenchée par la pression exercée par le cotexte : elle relève donc de la pragmatique. 


\section{Les CONTRIBUtions}

Dans cette présentation, nous avons essayé de montrer que des problématiques multiples - s'étendant du cœur de la grammaire jusqu'aux métaphores - peuvent être étudiées fructueusement à travers le prisme de multiples formes de conflit. Ces problématiques, bien entendu, ne peuvent pas être épuisées dans un numéro de revue. Dans ce volume, la notion de conflits est donc le centre à partir duquel les contributions s'étendent en éventail, en développant des aspects variés.

M. Prandi $(1992,2004)$ propose un style de recherche qu'il appelle « grammaire philosophique ». L'idée est que, pour comprendre le fonctionnement de la langue, il est nécessaire de postuler un fond de contenus plus stable de toute langue ou culture: la nature de ces contenus n'est ni sémantique, ni cognitive, mais ontologique. A partir de là, l'enjeu est double : d'une part, il s'agit de délimiter ce qui relève de cette ontologie et ce qui relève du code linguistique ; de l'autre, il s'agit d'étudier les interactions entre ces niveaux. Dans les deux cas, les conflits sont les phénomènes les plus révélateurs.

M. Fasciolo utilise l'étude des conflits conceptuels pour s'interroger sur les interactions entre lexique et ontologie .

R. Trim s'interroge sur le rapport entre l'étude des tropes dans tradition cognitive et l'étude des tropes dans la grammaire philosophique proposée par Prandi (1992, 2017). Paissa et Rossi étudient des métaphores littéraires et des langues de spécialité à travers l'équilibre entre cohérence et conflit conceptuel.

Enfin, G. Kleiber aborde une problématique plus périphérique : la relation entre le sens non conflictuel ou « littéral » et le sens conflictuel ou " figuré » dans les expressions et les proverbes métaphoriques (couper les ponts vs. l'habit ne fait pas le moine).

Notre objectif est que l'hétérogénéité des contributions, tout comme notre présentation, mette en lumière le fait que la notion de conflit est suffisamment générale pour offrir une vision organique des phénomènes très variés, et, en même temps, suffisamment ductile pour permettre de saisir la spécificité de chacun. 


\section{Références}

CARNAP, R. (1932 [2010]), «Le dépassement de la métaphysique par l'analyse logique du langage », in Soulez (éd.), Manifeste du Cercle de Vienne et autres écrits, Paris, Vrin, 149-171.

Chomsky, N. (1957[1979]), Structures syntaxiques, Paris, Éditions du Seuil.

Chomsky, N. (1965[1971]), Aspects de la théorie syntaxique, Paris, Éditions du Seuil.

Cruse, D. A. (1986), Lexical Semantics. Cambridge, Cambridge University Press.

Cohen, J. (1979), « Théorie de la figure » dans Todorov, T. et al. (éd): Sémantique de la poésie. Paris, Éditions du Seuil, 84-127.

DIK, S. (1989 [1997]), The Theory of Functional Grammar. Part I : The Structure of the Clause, Dordrecht-Providence. $2^{\text {ème }}$ éd. : Mouton De Gruyter, Berlin - New York.

DuCrot, O. (1980), « Note sur la polyphonie et la construction des interlocuteurs », dans: Ducrot, O et al.: Les mots du discours, Paris, Les Éditions de Minuit, 233236.

FASCIOLO, M. (2016), « Des métaphores de la vie quotidienne à l'ontologie de la vie quotidienne », Langue Française, 189, 49-65.

FASCIOLO, M. (2018), «Les noms du fonds, ou la fonction des noms dits "sommitaux"», in Franck Neveu dir., avec la collab. de Bernard Harmegnies, Linda Hriba, Sophie Prévost : Actes du 6e Congrès Mondial de Linguistique Française. Mons, 9-13 juillet 2018. ILF, Paris, EDP Sciences

FAsciolo, M. \& Rossi, M. (2016), « Métaphore et métaphores: les multiples issues de l'interaction conceptuelle », Langue Française, 189, 5-12.

Fillmore, CH. (1968), "The case for case", dans E. Bach, R. Harms (éd.), Universals in Linguistic Theory. New York, Holt, Rinehart \& Winston, 1-88.

Fillmore, CH. (1977), "The case for case reopened”, dans P. Cole \& J. M. Sadock (éd), Syntax and Semantics 8, 59-81.

FONTANIER P., (1821 [2009]), Les Figures du discours, Paris : Flammarion.

Francis E. J. \& Michaelis A. L. (2003), Form-Function Incongruity and the Architecture of Grammar. Standford, CSLI.

Genette G. (1966), Figures, Paris, Éditions du Seuil.

Goldberg, A. E. (1995), Constructions: A Construction Grammar Approach to Argument Structure, Chicago, Chicago University Press.

GROUPE $\mu$ (1970), Rhétorique générale, Paris, Larousse.

HALlyn, F. (1975), Formes métaphoriques dans la poésie lyrique de l'âge baroque en France. Genève, Droz.

Hallyn, F. (1987), La structure poétique du monde : Copernic, Kepler. Paris, Editions du Seuil.

HAIMAN, J. (1985), "Introduction", in J. Haiman (éd.), Iconicity in Syntax, Amsterdam - Philadelphie John Benjamins, 1-7.

HANKs, P. (2013), Lexical Analysis. Cambridge MA, MIT Press. 
JAKobSOn, R. (1966), "A la recherche de l'essence du langage », Diogène 51, Problèmes du langage, 22- 38.

KleIBer, G. (1999), Problèmes de sémantique : la polysémie en questions, Sens et structures, Villeneuve d'Ascq, Presses Universitaires du Septentrion.

LANGACKER, R. (1993), "Clause structure in cognitive grammar", Studi italiani di linguistica teorica e applicata XXII : 465-508.

LANGACKER, R. (2000), Grammar and conceptualisation. Berlin, Mouton de Gruyter.

LAKOFF, G. \& M. JOHNSON (1980[1986]), Les métaphores dans la vie quotidienne. Paris : Minuit.

Moens, M. \& M. STEedman (1988), “Temporal ontology and temporal reference”. Computational. Linguistics, $14 / 15$ en ligne.

NiCOLAS, D. (2002), La distinction entre noms massifs et noms comptables: aspects linguistiques et conceptuels. Louvain, Editions Peeters.

PERrin, L. (1996), L'ironie mise en trope, Paris : Éditions Kimé.

Pusteyovsky, J. \& E. JeZEK (2008), «Semantic coercion in language: Beyond distributional analysis », Italian Journal of Linguistics 20(1), 181-214.

Prandi, M. (1987), Sémantique du contresens. Essai sur la forme interne du contenu des phrases. Paris, Les Éditions de Minuit.

PRANDI, M. (1992), Grammaire philosophique des tropes. Mise en forme linguistique et interprétation discursive des conflits conceptuels. Paris, Les Éditions de Minuit.

Prandi, M. (2000), « Littéral, non littéral, figuré », Cahiers de praxématique, 35, 17-38.

PRANDI, M. (2004), The Building Blocks of Meaning, Amsterdam - Philadelphie John Benjamins.

PRANDI, M. (2016), "Selection Restrictions as Ultimate Presuppositions of Natural Ontology", Topoi, 35, 73-81.

Prandi, M. (2017), Conceptual Conflicts in Metaphors and Figurative Language, , New York - Londres, Routledge.

RABAtel, A. (2008) (éd.), Figures et points de vue, Langue Française, 160.

SPERBER, D. \& Wilson, D. (1986[1989]), La pertinence. Communication et Cognition, Paris, Les Éditions de Minuit.

STEEN G. J. (2008), “The Paradox of Metaphor : Why we Need a Three-dimensional Model for Metaphor", Metaphor and Symbol, 23 (4), 213-241.

STEEN, G. J. (2011), "The contemporary theory of metaphor - now new and improved". Review of Cognitive Linguistics 9 (1), 26-64.

TESNiÈre, L. (1959[1966]), Eléments de syntaxe structurale, $2^{\text {ème }}$ éd., Paris, Klincksieck.

Wittgenstein, J. J. L. (1921[1972]), Tractatus logico-philosophicus. Paris, Gallimard. 